\title{
Wer hat Angst vor dem Bösen Wolf?
}

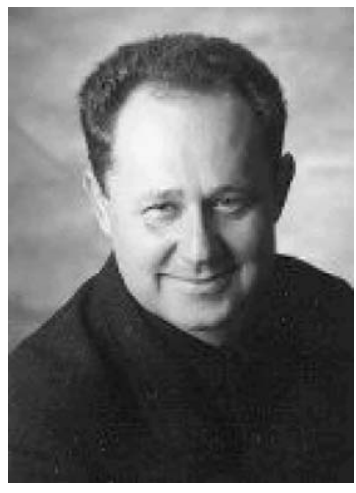

Prof. Dr. med. Manfred Wildner
Die Regeln für das Kinderspiel „Wer hat Angst vor dem Bösen Wolf?" sind denkbar einfach: Ein Kind, welches sich als böser Wolf zur Verfügung stellt, steht den mitspielenden Kindern mit etwas Abstand gegenüber. Der böse Wolf ruft dann: „Wer hat Angst vor dem Bösen Wolf?“ Worauf hin die Kinder antworten: „Niemand!“ Darauf hin fragt der böse Wolf weiter: „Und wenn er kommt?“ Die Kinder antworten: „Dann laufen wir davon!“. Die Mitspieler versuchen nun, sich so schnell wie möglich an einem zuvor vereinbarten Ort in Sicherheit zu bringen. Der böse Wolf versucht, diese auf dem Weg dorthin zu fangen. Die gefangenen Kinder müssen ihm dann bei der nächsten Runde helfen (www.kinder.de/ Bewegungsspiele).

Richtig Angst hat wohl niemand dabei. Grundelement dieses Spieles ist ein eher spielerischer Umgang mit dem Gefühl „Angst“. „Furcht hat man, Angst hat Einen“ lautet ein Merksatz der Psychiatrie. Ausgedrückt wird damit die Unterscheidung zwischen dem gegebenen Objektbezug der Furcht, welcher sich konkretisieren lässt, und dem objektunbestimmten Gefühl der Angst. Angst mit seinem ursprüngliche Bedeutungsfeld von Enge, Beengung, Bedrängnis ist ein sprachlicher Exportschlager: im Englischen findet man die „German Angst" bereits im 19. Jahrhundert als Bezeichnung für eine Art Existenzangst.

Der Umgang mit der Angst will offensichtlich gelernt sein. Das Spiel mit dem „bösen Wolf“ ist dem Märchen von Rotkäppchen entlehnt, in Frankreich (Le Petit Chaperon rouge, Charles Perrault, 1697) wie in Deutschland (Rothkäppchen, Brüder Grimm, 1812) gleichermaßen bekannt. Das spielerische Kennenlernen auch gefährlicher und bisweilen grausamer Seiten des Lebens und das damit verbundene Erlernen einer ersten Risikokompetenz ist wohl eine der Funktionen von Märchen. „Kinder brauchen Märchen", so die Ansicht des Psychoanalytikers Bruno Bettelheim, für den das Motiv des finsteren Waldes für „die dunkle, verborgene, fast undurchdringliche Welt unseres Unbewussten“ steht, welches es auf dem Weg zum eigenen Ich zu erobern gilt [2]. Psychoanalytisch werden auch „Grundformen der Angst“ (Riemann, [1]) unterschieden: die Angst vor dem Ich-Verlust der Selbsthingabe, die Angst vor der Ungeborgenheit der Selbst-Werdung, die Angst vor der Unsicherheit von Wandlung und die Angst vor der Unfreiheit von Notwendigkeiten. Inwieweit diese Unterscheidung empirisch bzw. auch neurowissenschaftlich belegbar ist, sei dahingestellt.

Angst und Furcht haben eine wichtige schützende Funktion, welche sich evolutionsbiologisch erklären lässt: Sie sind der psychologische und physiologische Zustand, welcher den wichtigen
Schutzmechanismen „Flucht“ und „Kampf“ vorausgeht und unsere Urahnen durch die geschichtlichen und vorgeschichtlichen Zeiten wohl so manches Mal erfolgreich vor Bedrohungen bewahrt bzw. aus bedrohlichen Situationen gerettet hat. Bei allen von uns gilt: Wir sind die Nachkommen der Überlebenden. Dass dieser Schutzmechanismus einer ganzen Reihe von Fehlalarmen unterliegt, liegt in der Natur der Sache: lieber 10 Mal Fehlalarm als einmal tot.

Bezogen auf eine einzelne Person lassen sich typische physiologische Reaktionen beschreiben, welche als Kampf- oder Fluchtreaktionen (fight or flight) gedeutet werden: Erhöhte Aufmerksamkeit, angespannte Muskeln, erhöhter Blutdruck und erhöhte Herzfrequenz, schnellere Atmung und anderes mehr. Bluthochdruck in diesem Sinne als unangemessene Reaktion in einer zivilisierten Lebenswelt mit nur mehr verkürzten körperlichen Kampf- und Fluchtritualen: Vielleicht hat das anfangs vorgestellte Spiel „Wer hat Angst vor dem Bösen Wolf?" vor diesem Hintergrund ja durchaus therapeutische bzw. präventive Dimensionen?

Von gleicher Bedeutung wie der individuelle Umgang mit Angst ist die Betrachtung von Angst als Gruppenphänomen. Tatsächlich finden sich hier ebenfalls interessante eigenständige Reaktionsmuster. Sie reichen von Xenophobie, also der Angst vor dem und den Fremden, über ohne erkennbaren naturwissenschaftlichen Grund auftretende Gruppenphänomene wie Ohnmachtsanfälle oder Erbrechen, welche auch als mass sociogenic illness bezeichnet werden, bis zur Massenpanik $[3,4]$. Offenbar können diese Befindlichkeiten in sozialen Gruppen von Person zu Person weitergegeben werden, so wie die schon eingefangenen Kinder beim Spiel vom Bösen Wolf mithelfen, die anderen Kinder auch noch zu fangen. Gibt es Gegenmittel? Neben der eingangs beschriebenen kommunikativen „Desensibilisierung“ ist in einer Krise ein vorbereitetes Schadensaufwuchskonzept ein wichtiger Baustein zur Krisenbewältigung [5]. Außerhalb einer akuten Krise sind Risikoanalyse, Risikobewertung und Risikokommunikation wichtige Bestandteile eines effektiven Risikomanagements, insbesondere im öffentlichen Raum: „Gefahr erkannt, Gefahr gebannt". Gemeint ist die konkrete Aufarbeitung einer erkannten Gefährdung, verbunden mit der Vergabe von spezifischen Bezeichnungen und einer Quantifizierung von Schadenswahrscheinlichkeiten. Auch diese Handlungskompetenz durch das Wissen um den richtigen Namen oder ein Zauberwort ist ein bekanntes Märchenmotiv: „Ach wie gut, dass niemand weiß, dass ich Rumpelstilzchen heiß'“ - Angst kann nur so lange verbreitet werden, bis eben dieser Name bekannt ist. 
In der wissenschaftlichen Beschäftigung mit Risiken wird begrifflich differenziert: Zwischen dem Schaden selbst, welcher die gesundheitlichen Auswirkungen beschreibt, der Gefahr, welche eine inakzeptabel hohe Schadenseintrittswahrscheinlichkeit beschreibt, dem Risiko als Funktion von Schadenspotenzial und Eintrittswahrscheinlichkeit. Durch Vorsorgemaßnahmen soll die Gefahr, also die Schadenseintrittswahrscheinlichkeit, auf ein akzeptables Maß gesenkt werden, das Restrisiko.

Zur Beschreibung dieses Restrisikos wurden z. B. in der Toxikologie verschiedene Begriffe in Form von Akronymen entwickelt „Zauberworte“ für Nicht-Experten: ALARA (ein Risiko as low as reasonable achievable, also so niedrig wie vernünftigerweise erreichbar), ALATA (ein Risiko as low as technically achievable, also ein technisch machbarer Wert), ADI (der acceptable daily intake, akzeptable tägliche Aufnahme), TDI (der tolerable daily intake, tolerierbare tägliche Aufnahme). In der Regel liegen diesen Werten Toxizitätsstudien mit Tieren zu Grunde, für welche die Effektgrenzen um den Faktor 10 für Variabilitäten der Empfindlichkeit zwischen biologischen Arten und nochmals um den Faktor 10 für Unterschiede der Empfindlichkeit einzelner Individuen erhöht werden.

Und die Realität hinter unseren Kinderspielen und unserem erwachsenen und ernsthaften Risiko- und Krisenmanagement? „Böse Wölfe“ existieren. Sie erzeugen zumeist mehr Angst bei Kindern als bei Erwachsenen bzw. Experten. Warum dies so ist? Hier kann angeführt werden, dass Erwachsene bzw. Experten über eine größere Handlungskompetenz verfügen, sich also wehren bzw. die Situation besser einschätzen können. Und zudem um die Möglichkeiten der medizinischen Versorgung im Schadensfall wissen. All dies fehlt den Kindern. Umgekehrt können Heranwachsende auch eine unangemessene Sorglosigkeit im Umgang mit Gefahren an den Tag legen: im Straßenverkehr, beim Sport, bei der ersten Zigarette. Dabei sind Erwachsene nicht generell die besseren Risikomanager.

Wie steht es mit der Risikokompetenz des Gesundheitswesens? Ist auch hier vernünftige Gelassenheit im Umgang mit unvermeidbaren Restrisiken zu finden, vergleichbar der Handlungs- und Risikokompetenz Erwachsener bezogen auf Wölfe? Oder befinden wir uns hier oftmals in der Situation aufgeregter Kinder, mit aktivierten Alarmsystemen angesichts unbekannter oder Bedrohungen bzw. als nicht einschätzbar empfundener Gefahrensituationen? Und umgekehrt: Erkennen wir auch den „Wolf im Schafspelz“, die verschleierte Gefahr, die Anpassung des Grenzwerts an ökonomische Interessen, den zuweilen raffinierten Denialismus in der gesellschaftlichen Kommunikation von Risiken? Wissen wir, dass der Straßenverkehr 10-mal so häufig tötet wie Gewaltverbrechen, Alkohol 20 Mal so häufig, geringer Obst- und Gemüseverzehr 50 Mal so häufig, Tabak und Bluthochdruck 200 Mal so häufig? Dass wir uns auf Urlaubsreisen in warmen Zonen mehr vor (herab fallenden) Kokosnüssen in acht nehmen müssen als vor Haien, vor allem aber vor Verkehrsunfällen? Wie viele zusätzliche Todesfälle das Vermeiden von Flugzeugen nach den Terroranschlägen des 11. September 2001 gefordert hat?

Diese Aspekte haben durchaus auch Bedeutung für das Gesundheitswesen. Sie bestimmen die Entscheidungen bei der Zuordnung von Ressourcen mit: Innerhalb der therapeutischen Medizin und auch zwischen präventivmedizinischen und therapeutischen bzw. rehabilitativen Handlungsoptionen. Diese Fragen wären irrelevant, stünden unbegrenzte Ressourcen zur Verfügung. Angesichts begrenzter Ressourcen bedeutet jede positive Förderentscheidung gleichzeitig ein Nein zu einer Reihe von konkurrierenden Handlungsoptionen. Unstrittig ist, dass in der Realität eine Reihe von suboptimalen Entscheidungen getroffen und Über-, Unter- und Fehlversorgung als Normalitäten des
Gesundheitswesens gelebt wurden und werden. Belegt ist auch, dass die Risikokompetenz in der allgemeinen Bevölkerung wie in den Gesundheitsberufen eher gering ist [6]. Anstrengungen für eine Verbesserung werden innerhalb des Gesundheitswesens z. B. im Rahmen der Global Burden of Disease Studie unternommen [7]. Der Risikoforscher Gerd Gigerenzer legt nahe, eine allgemeine bevölkerungsweite Risikokompetenz auch mithilfe pädagogischer Interventionen nachdrücklich zu fördern, um so zu einer gesünderen Gesellschaft mit besseren Allokationsentscheidungen zu gelangen (http://www.harding-center.de).

Möglicherweise erst um den Preis des Lebens zu erwerben ist eine Lebensklugheit und gereifte Gelassenheit, welche die große „Chance Leben“ nicht dem verzerrtem Bild eines allgegenwärtigen übermächtigen „Lebens-Risiko“ opfert. Gegenpole der Angst sind Vertrauen und vertrauensvolle Kommunikation, sozialer Zusammenhalt insgesamt, Beruhigung durch das Wissen über verantwortungsvoll und kompetent getroffene Vorsorge- und Handlungskompetenzen. Im Salutogenesekonzept von Aaron Antonovsky findet man all dies wieder - nicht zufällig. In diesem Sinne möchte auch diese Ausgabe der Zeitschrift zu einem kompetenten Umgang mit Risiken und Chancen im Gesundheitswesen beitragen: mit Artikeln zu den Entscheidungen des Gemeinsamen Bundesausschusses (G-BA), zur Früherkennung des Prostatkrebses mit PSA-Testung, zum Resident Assessment Instrument in der Pflege, zur Führung von Krankenhausallianzen, zu den Rauchgewohnheiten von Mitarbeitern im Gesundheitswesen, zu Ergebnissen zum regionalen Tabakkonsum aus dem Mikrozensus, zur Gesundheitsförderung bei allergischen Erkrankungen in Grundschulen und zur körperlichen Leistungsfähigkeit bei kardiovaskulären Erkrankungen.

Wer hat Angst vor dem Bösen Wolf? Wir alle sollten Respekt vor Wölfen haben. Dabei sollte angemessener Respekt nicht dazu führen, den Wald nicht mehr zu betreten oder alle vermeintlich bösen Wölfe zu töten - was im Umgang mit vielen Risiken ohnehin ausgeschlossen ist. Und stimmt der Begriff des „bösen“ Wolfes überhaupt, im Licht von Artenschutz und Achtsamkeit gegenüber einem Netz des Lebens und seinen natürlichen und auch zivilisatorischen Grundlagen? Ist des Menschen Natur nicht auch seine Kultur? Angst ist ein schlechter Ratgeber. Nicht die vollständige Elimination ist in der Regel der „sichere Ort“, an den wir uns flüchten können, sondern die auch wissenschaftliche Aufarbeitung, der auch gesellschaftlich gelernte Umgang mit einem Restrisiko und - um beim Bild zu bleiben - auch dass Einüben einer friedlichen Koexistenz, gekennzeichnet durch gereiftes Bewusstsein um Risiken und Chancen, Respekt von Grenzen und Schutz von Lebensräumen. Dann brauchen wir vor nichts mehr Angst zu haben - außer vor der Angst (Franklin D. Roosevelt).

\section{Literatur}

1 Riemann F. Grundformen der Angst und die Antinomien des Lebens. Ernst Reinhardt; Basel/München: 1961

2 Bettelheim B. Kinder brauchen Märchen. Deutsche Verlags-Anstalt; Stuttgart: 1977

3 Jones TF, Craig AS, Hoy D et al. Mass psychogenic illness attributed to toxic exposure at high school. N Engl J Med 2000; 342: 96-100

4 Wessely S, Hyams KC, Bartholomew R. Psychological implications of chemical and biological weapons. Br Med J 2001; 323: 878-879

5 Bundesamt für Bevölkerungsschutz und Katastrophenhilfe. Biologische Gefahren I. (3. Auflage) BBK; Bonn: 2007

6 Gigerenzer G, Muir Gray JA, Hrsg. Better Doctors, Better Patients, Better Decisions. Envisioning Health Care 2020. Cambridge Massachusetts: MIT Press; 2011

7 Lim SS, Flaxman AD, Danaei $G$ et al. A comparative risk assessment of burden of disease and injury attributable to 67 risk factors and risk factor clusters in 21 regions, 1990-2010: a systematic analysis for the Global Burden of Disease Study 2010. Lancet 2012; 380: 2224-2260 